\title{
Connected Capacitive Sensor Array for Upper-Extremity Motor Rehabilitation
}

\author{
Haoyan Liu \\ University of Arkansas \\ Fayetteville, Arkansas \\ hl002@uark.edu
}

\author{
James P. Parkerson \\ University of Arkansas \\ Fayetteville, Arkansas \\ jparkers@uark.edu
}

\author{
Alexander Nelson \\ University of Arkansas \\ Fayetteville, Arkansas \\ ahnelson@uark.edu
}

\begin{abstract}
This paper proposes a rehabilitation assitant system equipped with a capacitor sensor array (CSA) for persons exhibiting upper-extremity motor impairments. The CSA utilizes mutual capacitance to quantize patients' hand motions on a rehabilitation activity board. The rehabilitation board is equipped with an accelerometer to detect the slope of the activity board, since different inclinations of motions may affect the motor quality post trauma. A microcontroller - a Texas Instruments MSP430FR2633 - is used to measure capacitance. A secondary microcontroller - an ATmega 328P with the Arduino firmware - is used to retrieve capacitive sensor and accelerometer data, and transfer data via Bluetooth to a web capable edge device. The edge device hosts a webpage displaying the patient's hand positions and the slope position of the activity board to enable visual feedback during and after rehabilitation sessions.
\end{abstract}

\section{CCS CONCEPTS}

- Computer systems organization $\rightarrow$ Sensors and actuators; Embedded systems; • Applied computing $\rightarrow$ Consumer health;

\section{KEYWORDS}

capacitive sensors, upper-extremity motor rehabilitation, wireless health monitoring, gesture recognition

\section{ACM Reference Format:}

Haoyan Liu, James P. Parkerson, and Alexander Nelson. 2018. Connected Capacitive Sensor Array for Upper-Extremity Motor Rehabilitation. In Proceedings of ACM/IEEE International Conference on Connected Health: Applications, Systems and Engineering Technologies (CHASE'18). ACM, New York, NY, USA, 2 pages. https://doi.org/10.1145/3278576.3278584

\section{INTRODUCTION}

More than a million individuals in the U.S. are hospitalized annually due to brain injuries, spinal cord injuries, and new or recurring strokes $[1,4,6]$. Trauma as a result of stroke or physical injury typically involves a long-term rehabilitation process. The main purpose of this research is to re-engineer a rehabilitation activity board to construct a low cost and portable rehabilitation system that benefits

Permission to make digital or hard copies of all or part of this work for personal or classroom use is granted without fee provided that copies are not made or distributed for profit or commercial advantage and that copies bear this notice and the full citation on the first page. Copyrights for components of this work owned by others than the author(s) must be honored. Abstracting with credit is permitted. To copy otherwise, or republish, to post on servers or to redistribute to lists, requires prior specific permission and/or a fee. Request permissions from permissions@acm.org.

CHASE'18, September 26-28, 2018, Washington, DC, USA

(c) 2018 Copyright held by the owner/author(s). Publication rights licensed to ACM. ACM ISBN 978-1-4503-5958-0/18/09..\$15.00

https://doi.org/10.1145/3278576.3278584

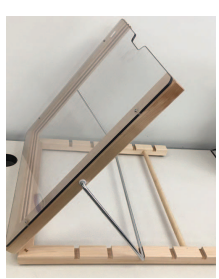

(a)

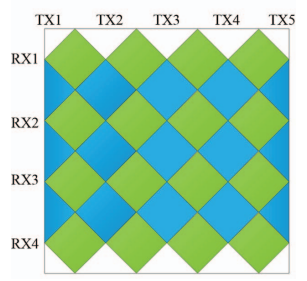

(b)
Figure 1: (a) Rehabilitation activity board with wood frame and plexiglass surface. (b) A $5 \times 4$ single pane differential capacitor sensor array topology.

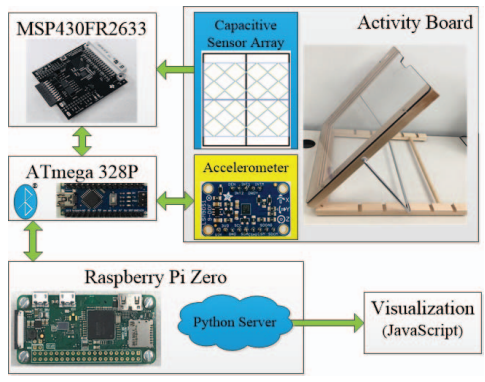

Figure 2: A block diagram of the rehabilitation assistant system.

both the rehabilitation facility and patients. A picture of the original rehabilitation activity board is shown in Figure 1a. Indium tin oxide (ITO) films are widely used for capacitive touch screen panel fabrications due to its transparency, low cost, and conductivity [2]. Those characteristics make ITO a perfect candidate to construct a low cost mutual CSA. In this project, a microcontroller - a Texas Instruments MSP430FR2633 - is used to sense the capacitance change when a touch occurs on the activity board. Existing literature has investigated various design patterns of capacitive touch sensors such as diamond-shaped pattern, snowflake-shaped pattern, and fork-shaped pattern $[2,3]$. In this initial prototype of the project, a conventional single layer diamond-shaped pattern is adopted and investigated. The single layer 4 X 5 capacitive sensor array, shown in Figure 1b, is created with the transparent ITO material.

In addition to sensing capacitance on the rehabilitation board, the slope of the board is also monitored. Post trauma, a patient may experience trouble in performing certain movements on the equipment when the slope of the activity board rises more sharply. The slope detection of the rehabilitation equipment is determined using a 3-axis accelerometer on the surface of the activity board. 
The MSP430 scans the sensor array and measures the capacitance at geometrically distributed points. A host microcontroller is used to retrieve capacitive sensor data from the MSP430 through I2C and obtain angle readings from the accelerometer. After processing, the data is transmitted to a Linux-based device, Raspberry Pi (RPi), via Bluetooth. An overview of the system is given in Figure 2 . This paper is organized in four sections. The following section discusses the implementation of the proposed system, followed by some preliminary test results of the system. The last section will conclude the paper and discuss the future work.

\section{IMPLEMENTATION}

For this prototype, the mutual capacitive sensor array is constructed with ITO-coated polyethylene terephthalate (PET) films. The sheet resistance of the PET films is 350-500 $\Omega / s q$ [8]. Both the transmitter and receiver lines of the capacitive sensors are built on the same layer to reduce cost. Each line of the transmitter and receiver sensors is electrically connected by copper tape. The copper tape currently facilitates conductivity to the extremes without having to propagate charge through the ITO PET. In the future, laser ablation will be considered to remove conductive material with high precision. We choose four lines of receiver (RX) electrodes in the sensor design to utilize all of the parallel receive measurements that the MSP430 can process simultaneously [7].

The microcontroller senses the capacitance changes of the sensor array when proximity or touch occurs in arbitrary units called "counts", with a scan rate of $50 \mathrm{~Hz}$. Change in the "counts" with respect to long-term average (LTA) value of each sensor can be identified as a touch or proximity event, specified at a location. The trade-off in the scan rate selection is between the fast scan response and low power consumption [7]. The data collected by the Arduino is converted to RGB values for visualization, and transmitted to the $\mathrm{RPi}$. The RPi hosts a web server written in Python. The RGB values of the sensors displayed on the website are updated by javascript. The color density visualizes the capacitance created by the hand above the array.

\section{RESULTS}

During a rehabilitation session, a patient may perform swipe-like motions horizontally, vertically, or diagonally on a rehabilitation board. A swipe upwards gesture is performed on a revised rehabilitation activity board, shown from left to right, on the top of Figure 3. The hand moves above the second and third columns of the CSA. Corresponding screen captures of the javascript frontend are shown on the bottom of Figure 3. The visualization results reflect the hand positions on the activity board. Two swipe gestures are tested on the sensor array. The author performed five upward swipes and five downward swipes, with results reported in Table 1. The system captured all the downwards swipes and four out of five upwards swipes. The activity board slope position is obtained by an accelerometer. During the test, the rehabilitation board is set horizontally on the test bench.

\section{CONCLUSION AND FUTURE WORK}

The prototype system is able to detect proximity, touch, and hand motions. Multi-touch is supported, which enables the system to
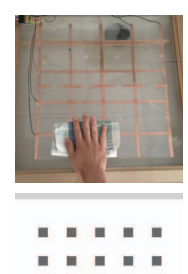

.1.

- . - . -
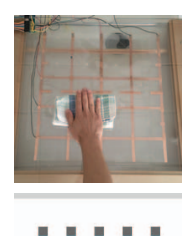

$-1$.

- .".

.1.....

- . - .

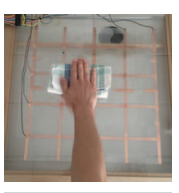

..".

-

...".

- . - .

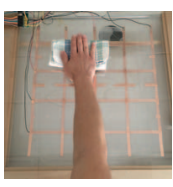

- !. - .

-

...".

...".
Figure 3: A depiction of a swipe up motion performed on the activity board, with javascript frontend showing calculated location at each frame.

Table 1: Gesture Recognition Results

\begin{tabular}{lrr}
\hline Gesture & \# Performed & \# Recognized \\
\hline Swipe Down & 5 & 5 \\
Swipe Up & 5 & 4 \\
\hline
\end{tabular}

recognize complex hand movement trajectories. The future work involves applying hand gesture recognition algorithm published in [5] to detect the patient's rehabilitation motions. The ultimate goal of the project is to develop a sensing methodology for detecting the recovery progress of a patient by analyzing physical attributes of the motions. This will help reduce the workload of medical professionals at rehabilitation facilities, as well as curtail the cost of rehabilitation process for patients.

\section{ACKNOWLEDGMENTS}

The authors would like to thank Susan Koch Fager and Tabatha Sorenson from Madonna Rehabilitation Hospital in Omaha Nebraska for input on rehabilitation equipment and its use in facilities. This work was supported by NSF SCH-1407035.

\section{REFERENCES}

[1] E. J. Benjamin, S. S. Virani, and C. W. Callaway. 2018. Heart Disease and Stroke Statistics-2018 Update: A Report From the American Heat Association. Circulation 17, 12 (2018), 493.

[2] B. Cannon and C. Brennan. 2014. Electrostatic Simulation Methodology for Capacitive Touch-Screen Panels. 25th IET Conference Proceedings (January 2014), 216-220(4).

[3] S. H. Lee, J. S. An, S. K. Hong, and O. K. Kwon. 2018. A Highly Linear and Accurate Fork-Shaped Electrode Pattern for Large-Sized Capacitive Touch Screen Panels. IEEE Sensors fournal (2018), 1558-1748.

[4] National Spinal Cord Injury Statistical Center. 2018. Facts and Figures at a Glance. Univ. Alabama Birmingham, Birmingham, AL, USA.

[5] A. Nelson, G. Singh, R. Robucci, C. Patel, and N. Banerjee. 2015. Adaptive and Personalized Gesture Recognition Using Textile Capacitive Sensor Arrays. IEEE Transactions on Multi-Scale Computing Systems 1, 2 (April 2015), 62-75. https: //doi.org/10.1109/TMSCS.2015.2495100

[6] C. A. Taylor, J. M. Bell, M. J. Breiding, and L. Xu. 2017. Traumatic Brian InjuryRelated Emergency Department Visits, Hospitalization, and Deaths. MMWR. Surveillance Summaries 66, 9 (2017), 1-16.

[7] Texas Instruments. 2015. "MSP430FR263x, MSP430FR253x Capacitive Touch Sensing Mixed-Signal Microcontrollers". [Revised: June 2017].

[8] ThorLabs. [n. d.]. ITO-Coated PET Film. Retrieved June 04, 2018 from https: //www.thorlabs.com/newgrouppage9.cfm?objectgroup_id=9535 